\title{
Passive targeting of ischemic-reperfused myocardium with adenosine-loaded silica nanoparticles
}

This article was published in the following Dove Press journal:

International Journal of Nanomedicine

23 March 2012

Number of times this article has been viewed

\section{Michael Galagudza' \\ Dmitry Korolev' \\ Viktor Postnov ${ }^{2}$ \\ Elena Naumisheva ${ }^{2}$ \\ Yulia Grigorova ${ }^{3}$ \\ Ivan Uskov' \\ Eugene Shlyakhto'}

'Institute of Experimental Medicine, VA Almazov Federal Heart, Blood and Endocrinology Center, ${ }^{2}$ Chemical Faculty, St Petersburg State University, ${ }^{3}$ Department of Pathophysiology, IP Pavlov State Medical University, St Petersburg, Russian Federation
Correspondence: Michael Galagudza Institute of Experimental Medicine, VA Almazov Federal Heart, Blood and Endocrinology Center, Akkuratova Str 2, St Petersburg, Russian Federation

Tel +78I 24997035

Fax +78I 24997069

Email galagoudza@mail.ru
Abstract: Pharmacological agents suggested for infarct size limitation have serious side effects when used at cardioprotective doses which hinders their translation into clinical practice. The solution to the problem might be direct delivery of cardioprotective drugs into ischemic-reperfused myocardium. In this study, we explored the potential of silica nanoparticles for passive delivery of adenosine, a prototype cardioprotective agent, into ischemic-reperfused heart tissue. In addition, the biodegradation of silica nanoparticles was studied both in vitro and in vivo. Immobilization of adenosine on the surface of silica nanoparticles resulted in enhancement of adenosine-mediated infarct size limitation in the rat model. Furthermore, the hypotensive effect of adenosine was attenuated after its adsorption on silica nanoparticles. We conclude that silica nanoparticles are biocompatible materials that might potentially be used as carriers for heart-targeted drug delivery.

Keywords: silica nanoparticles, targeted drug delivery, myocardium, ischemia, reperfusion

\section{Introduction}

Myocardial infarction is a major cause of morbidity and mortality worldwide. The advent of reperfusion strategies such as thrombolysis and percutaneous coronary intervention has resulted in significant improvement in survival and prognosis in patients with myocardial infarction. Along with the apparent favorable effects, reperfusion therapy has brought to light the problem of irreversible reperfusion injury, which is usually lethal. ${ }^{1}$ According to different estimates, acute reperfusion injury in patients treated with primary percutaneous coronary intervention can increase myocardial infarct size by $20 \%-50 \% .^{2}$ Therefore, it follows that prevention and/or alleviation of myocardial ischemia-reperfusion injury is one of the most important goals of medical care. One of the promising approaches for reducing cardiac ischemia-reperfusion injury is ischemic postconditioning, a phenomenon of infarct size reduction caused by induction of repetitive brief episodes of ischemia during early reperfusion after a prolonged ischemic insult. ${ }^{3,4}$ In recent years, it has been shown both in the experimental and clinical settings that the infarct-limiting effect of ischemic postconditioning can be mimicked by administration of certain cardioprotective drugs at the time of myocardial reperfusion or just prior to it. ${ }^{5}$ This phenomenon has been termed "pharmacological postconditioning". However, it should be noted that the agents used for pharmacological postconditioning may have serious side effects when used at the doses required for infarct size limitation, which hinders their translation into the clinical realm. ${ }^{1}$ One solution to this problem might be direct delivery of cardioprotective drugs 
into the ischemic-reperfused myocardium. This approach, known as "targeted drug delivery", offers major advantages as compared with systemic drug distribution, ie, reduced toxicity owing to selective drug accumulation in the target area and increased therapeutic efficacy of the drug. ${ }^{6}$

There are two basic principles suggested for targeted drug delivery into affected tissue using nanosized carriers. The first is usually referred to as "passive targeting", suggesting that the drug-loaded nanocarrier is passively retained in areas with increased microvascular permeability. ${ }^{7,8}$ The second is known as "active targeting", which may result in an even greater gradient of drug concentration between the intact and injured tissue due to the specific interaction of the tissue-recognition ligand engrafted on the surface of the carrier and a unique disease marker expressed either on the cell membrane or inside the cell. ${ }^{9}$ This interaction results in accumulation of the drug-enriched nanocarrier in the tissue of interest with subsequent release of the drug during biodegradation of the particle coating.

Much of the work on targeted drug delivery has been in the field of cancer treatment. At present, there is some (albeit limited) published evidence that the ischemic heart can be actively and passively targeted with drug-loaded liposomes. ${ }^{10,11}$ Other nanoparticles, including inorganic ones, also hold promise for drug delivery targeted to the heart. We have previously shown that 6-13 nm silica nanoparticles are not toxic during acute intravenous administration and can also be readily functionalized by immobilization or chemical assembly approaches. ${ }^{12}$

Although recent research has made significant strides in clarifying the mechanisms of endogenous and pharmacological cardioprotection, we still do not have an "ideal" cardioprotective agent with a favorable safety and efficacy profile. There is now considerable evidence for involvement of adenosine in the mechanism(s) of ischemic postconditioning-induced cardiac protection. In particular, the protective effect of ischemic postconditioning in the isolated rabbit heart was abolished after administration of DPCPX, a selective adenosine $A_{1}$ receptor blocker. ${ }^{13}$ Genetic deletion of $A_{1}$ and $A_{2 a}$ receptors attenuated the cardioprotective effects of ischemic postconditioning. ${ }^{14,15}$ Furthermore, exogenous infusion of NECA, a nonselective $\mathrm{A}_{1} / \mathrm{A}_{2}$ receptor agonist, ${ }^{16}$ and CGS-21680, a selective $\mathrm{A}_{2 \mathrm{a}}$ receptor agonist, ${ }^{17}$ immediately after prolonged ischemia had a pronounced infarct-limiting effect in the isolated rat heart and in vivo rabbit model, respectively. Taken together, these experimental data strongly suggest that adenosine can exhibit a potent cardioprotective effect when administered at the onset of myocardial reperfusion. However, clinical use of adenosine for myocardial protection is limited because of side effects, such as hypotension and bradycardia.

The present study investigated the potential of silica nanoparticles for delivery of adenosine, a prototype cardioprotective agent, into the ischemic-reperfused heart. Only a passive targeting strategy could be used, so we compared the infarct-limiting and hemodynamic effects of free adenosine with those of adenosine immobilized on the surface of silica nanoparticles. Further, biodegradation of silica nanoparticles was studied both in vitro and in vivo.

\section{Materials and methods Characteristics of silica nanoparticles and adenosine immobilization}

Highly dispersed standard pyrogenic silica (Aerosil ${ }^{\circledR}$ A380, Vekton, St Petersburg, Russia) was used throughout the experiments. The surface area of the silica nanoparticles was determined using the Brunauer-Emmett-Teller method and averaged $170-380 \mathrm{~m}^{2} / \mathrm{g}$. The mean particle diameter varied from $6 \mathrm{~nm}$ to $13 \mathrm{~nm}$. Prior to preparation of the aqueous suspensions, the silica nanoparticles were sterilized at $180^{\circ} \mathrm{C}$ for 3 hours. Adsorption of adenosine onto the surface of the silica nanoparticles was achieved by mixing the suspension of silica nanoparticles in sterile apyrogenic $0.9 \%$ sodium chloride and adenosine solution in the same vehicle. The samples were then subjected to 10 minutes of sonication and left at $+4^{\circ} \mathrm{C}$ overnight.

Adsorption efficiency was determined by spectrophotometric measurement (UV-2450, Shimadzu, Japan) of residual adenosine concentration in the solution after completion of adsorption. For adsorption studies, $6.9 \mathrm{mg}$ of adenosine was dissolved in $1 \mathrm{~mL}$ of distilled water followed by the addition of $20 \mathrm{mg}$ of silica nanoparticles and left for different time intervals. After that, the volume of the sample was adjusted to $9 \mathrm{~mL}$ and centrifuged at $10000 \mathrm{~g}$ for 3 minutes. Centrifuged solution was further diluted and analyzed spectrophotometrically. The spectra were registered at $\lambda=260 \mathrm{~nm}$ in quartz cuvettes. The optical length was selected empirically to achieve an optical density of $0.1-1.0$ at maximal absorption $\left(v=38.6 \times 103 \mathrm{~cm}^{-1}\right.$, which corresponds to $\lambda=260 \mathrm{~nm}$ ).

\section{Biodegradation of silica nanoparticles}

Biodegradation of the silica nanoparticles was studied both in vitro and in vivo. When designing the experiments, we speculated that the nanoparticles were undergoing biodegradation due to gradual erosion of their surface, resulting in formation of water-soluble salts of silicic acid which 
are excreted by the kidney (Figure 1). Suspension of Aerosil A380 in Krebs-Henseleit buffer with an electrolyte composition similar to that of plasma with a final concentration of silica nanoparticles of $2 \mathrm{mg} / \mathrm{mL}$ and $\mathrm{pH} 7.4$ was used for the in vitro biodegradation study. The experiments were performed under continuous stirring in a $100 \mathrm{~mL}$ polytetrafluoroethylene container. The temperature of the media was maintained at $37^{\circ} \mathrm{C}$ by water jacketing. The samples were continuously gassed with carbogen $\left(95 \% \mathrm{O}_{2}\right.$ and $\left.5 \% \mathrm{CO}_{2}\right)$. Total incubation time was 15 hours. Samples were taken at 5-hourly intervals and analyzed for silicate content spectrophotometrically after reaction with molybdenum blue at a wavelength of $815 \mathrm{~nm}$ in $1 \mathrm{~cm}$ layer cuvettes.

In vivo silica nanoparticle biodegradation was studied in male Wistar rats weighing 200-250 g. The animals were fed regular chow, and water was available ad libitum. All experiments were performed in accordance with the Guide for the Care and Use of Laboratory Animals and approved by the local ethics committee. The animals were anesthetized with sodium pentobarbital, given intraperitoneally at a dose of $60 \mathrm{mg} / \mathrm{kg}$. The silica nanoparticles were infused intravenously at a dose of $2 \mathrm{mg} / \mathrm{mL}$ and a volume of $1 \mathrm{~mL}$, followed by sampling of the liver at one hour, and 10,20, and 30 days after infusion ( $n=3-6$ at each time point). Animals that received an intravenous infusion of $1 \mathrm{~mL}$ of vehicle $(n=8)$ served as controls. Liver samples were dried at $90^{\circ} \mathrm{C}$ for 24 hours to obtain a constant weight. The liver was chosen for sampling and analysis on the basis of previous biodistribution experiments showing maximal accumulation of silica in this organ. ${ }^{12}$ Quantitative analysis of silicon content within the samples was performed using atomic absorption spectroscopy. The product of mineralization obtained after drying was analyzed by atomic absorption spectrometry with electrothermic atomization and Zeeman correction. Silicon content within the product of mineralization was recalculated for the dry sample weight and expressed in $\mu \mathrm{g} / \mathrm{g}$.

\section{Biodistribution of silica nanoparticles with and without myocardial ischemia}

For investigation of passive heart-targeted drug delivery using silica nanoparticles, we first studied the biodistribution of unmodified nanoparticles in rats with regional myocardial ischemia-reperfusion injury. Male Wistar rats weighing 200-250 g were anesthetized with sodium pentobarbital $60 \mathrm{mg} / \mathrm{kg}$ and used throughout the experiments. The animals were randomly allocated to one of three groups, ie, controls ( $\mathrm{n}=5$, intravenous infusion of saline), animals treated with silica nanoparticles $(2 \mathrm{mg} / \mathrm{mL}, 1 \mathrm{~mL}$ intravenously) and a sham surgical procedure $(n=6)$, and animals treated with silica nanoparticles and myocardial ischemia-reperfusion injury $(n=5)$. Under mechanical ventilation, regional myocardial ischemia was induced by 30 minutes of left coronary artery ligation followed by 60 minutes of reperfusion. The silica nanoparticles were infused for 10 minutes, starting 5 minutes prior to reperfusion. The left ventricle of the heart and the liver were sampled at the end of the experiments, then rinsed and dried at $90^{\circ} \mathrm{C}$ for 24 hours to obtain a constant weight. Determination of the silicon content in the organ

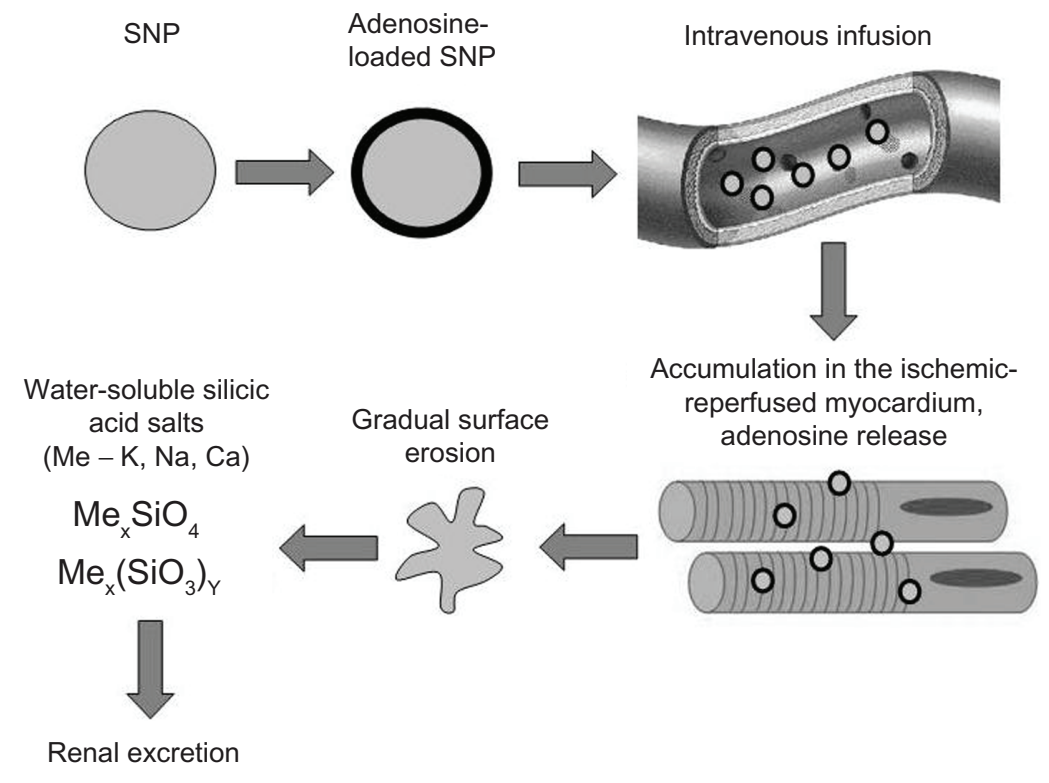

Figure I Proposed algorithm of passive heart targeting with silica nanoparticles. Abbreviation: SNP, silica nanoparticles. 
samples was done using atomic absorption spectroscopy as described above. Direct evidence of the effectiveness of targeted drug delivery can be obtained only by measuring drug concentration in target tissue. However, this is not feasible with adenosine, which has a half-life of less than 10 seconds in blood. ${ }^{18}$

\section{Hemodynamic effects of nanoparticle- bound and free adenosine}

Adenosine bound to silica nanoparticles was administered to the anesthetized rats intravenously for 10 minutes at a dose of $300 \mu \mathrm{g} / \mathrm{kg} / \mathrm{minute}(\mathrm{n}=6)$. Control animals received a 10-minute intravenous infusion of adenosine at a dose of $300 \mu \mathrm{g} / \mathrm{kg} / \mathrm{minute}(\mathrm{n}=8)$. Mean blood pressure was registered in the common carotid artery before the beginning of drug infusion, at minutes 5 and 10 of infusion, and at minutes 5 and 10 of recovery.

\section{Effects of nanoparticle-bound and free adenosine on infarct size}

Five minutes prior to the end of 30 minutes of ischemia, the anesthetized animals received either a 10-minute adenosine infusion at a dose of $300 \mu \mathrm{g} / \mathrm{kg} /$ minute $(\mathrm{n}=5)$ or an infusion of adenosine adsorbed onto silica nanoparticles at the equivalent dose $(n=6)$. Control animals were treated with vehicle $(n=8)$. The left coronary artery was reoccluded after 90 minutes of reperfusion, followed by administration of $0.5 \mathrm{~mL}$ of 5\% Evans Blue (MP Biomedicals, Santa Ana, CA) via the femoral vein for identification of the area at risk. The hearts were excised and cut into five $2 \mathrm{~mm}$ thick slices parallel to the atrioventricular groove. The basal surface of each slice was digitally photographed. The slices were immersed in a $1 \%$ solution of 2,3,5-triphenyltetrazolium chloride at $37^{\circ} \mathrm{C}(\mathrm{pH} 7.4)$ for 15 minutes and photographed again for identification of infarct area. The images were digitized using Adobe Photoshop $\mathrm{CS}$. The area at risk was expressed as a percentage of the whole slice, and the infarct area was expressed as a percentage of the area at risk. Values of area at risk and infarct area for each heart were obtained by summarizing data for the slices and calculating mean values. Statistical analyses were performed using the nonparametric Mann-Whitney U test, with $P$ values $<0.05$ considered to be statistically significant.

\section{Results}

\section{Effectiveness of adenosine adsorption onto silica nanoparticles}

The adenosine content on the surface of the silica nanoparticles was $0.017 \mathrm{mmoL} / \mathrm{g}$, while adsorption efficiency was
$4.5 \mathrm{mg} / \mathrm{g}$. Complete adsorption of adenosine on the silica nanoparticle surface was achieved within 15 minutes.

\section{Biodegradation of silica nanoparticles in vitro and in vivo}

Results of analyses of samples obtained in the in vitro experiments are shown on Figure 2. As a rule, physical or chemical processes of this kind (eg, material aging, changes in relative surface area, dissolution) are asymptotic and might be adequately described by the following inverse polynomial of the second degree:

$$
D=a+b /(T+1)+c /(T+1)^{2}
$$

where $D$ is relative optical density, $T$ is time (days), and $a$, $b$, and $c$ are coefficients of the polynomial.

Because the relative optical density in the analytical method used is linearly correlated with the concentration of silicate ion, the changes of the latter could also be described by equation (1). In this case, changes in the silicate concentration with time in the samples investigated might be described by equation (1) with the following coefficients:

$$
\begin{gathered}
a=0.0224195 \\
b=-0.0479528 \\
c=0.0255494
\end{gathered}
$$

Coefficient $a$ is the maximal theoretically possible relative optical density in this process and hence is proportional to the maximal concentration of silicate. Using inverse recomputation according to equation (1), it is possible to derive the value which corresponds to $95 \%$ biodegradation of silica, which is 41 days. These data fit well with the results of Finnie et $\mathrm{al}^{19}$ who studied biodegradation of sol-gel mesoporous silica microparticles and showed that silica microparticles

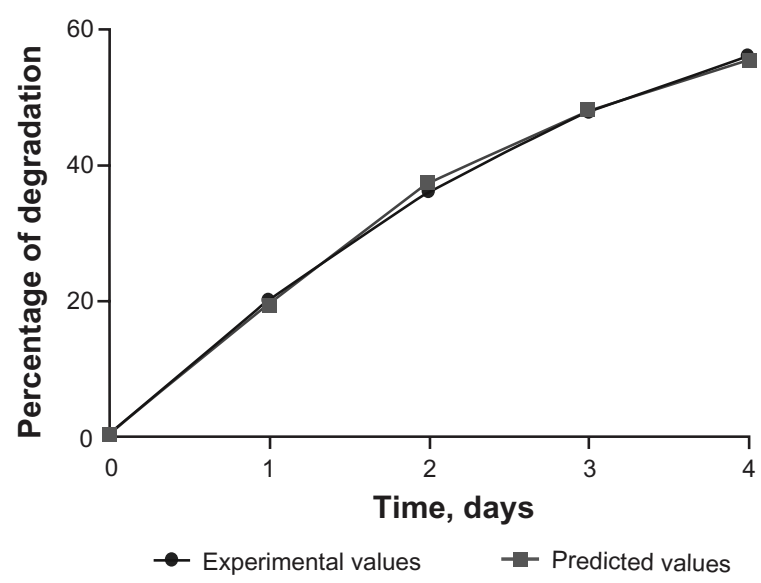

Figure 2 Relationship between silica concentration and time in in vitro biodegradation experiments. 
are rapidly degraded in physiological buffer. Further, the authors demonstrated that addition of plasma proteins to the buffer retarded biodegradation by $20 \%-30 \%$. It follows that biodegradation in vivo might be slower than in the in vitro setting.

With regard to the in vivo biodegradation data, the silicon content in the liver at different times after intravenous silica nanoparticle infusion is shown in Figure 3. The normal silicon content in the liver of control rats averaged $4.4 \pm 1.55 \mu \mathrm{g} / \mathrm{g}$. At one hour after silica nanoparticle infusion, the silicon content increased up to $282.3 \pm 62.65 \mu \mathrm{g} / \mathrm{g}$. Ten and 20 days after infusion, the silicon content in the liver was significantly lower $(81.5 \pm 9.25$ and $30.2 \pm 7.48 \mu \mathrm{g} / \mathrm{g}$, respectively). These dynamics of silica nanoparticle dissolution are consistent with the data obtained in the in vitro model. However, at 30 days following silica nanoparticle administration, we did not see any additional decrease in liver tissue silicon content $(31.5 \pm 11.87 \mu \mathrm{g} / \mathrm{g})$, which might have been because of the higher stability of the intracellular pool of silica nanoparticles produced as a result of phagocytosis by Kupffer cells and, to a lesser extent, internalization of silica nanoparticles into hepatocytes and other liver cells. However, it remains unclear whether this increased silicon content in hepatic cells affects liver function or not.

\section{Biodistribution of silica nanoparticles}

In the controls, the silicon content was found to be significantly higher in myocardial tissue than in the liver (32 \pm 2.8 versus $4 \pm 2.3 \mu \mathrm{g} / \mathrm{g}$, respectively, $P<0.05$, Figure 4). The physiological process involved is currently unknown. Administration of silica nanoparticles to the sham-operated rats resulted in a dramatic rise in liver silicon content as compared with controls $(163 \pm 19.5$ versus $4 \pm 2.3 \mu \mathrm{g} / \mathrm{g}$, respectively, $P<0.01$ ), while the myocardial silicon concentration remained unaffected $(27 \pm 9.7$ versus $32 \pm 2.8 \mu \mathrm{g} / \mathrm{g}$, respectively, $P>0.05)$. Administration of

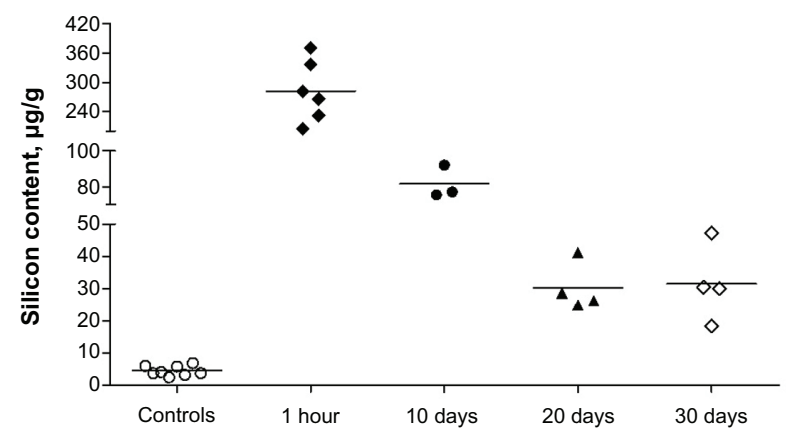

Figure 3 Biodegradation of silica nanoparticles in vivo: silicon content in the liver at different durations after intravenous infusion of silica nanoparticles.

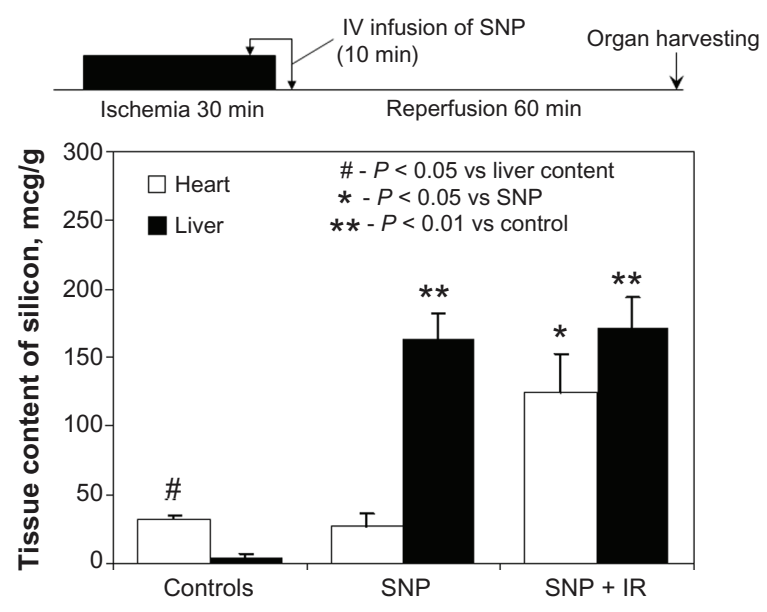

Figure 4 Biodistribution of nonmodified silica nanoparticles in a rat model of myocardial ischemia-reperfusion. Top, experimental protocol; bottom, silica content in the heart and liver samples.

Abbreviations: SNP, silica nanoparticles; IR, ischemia-reperfusionIV, intravenous.

silica nanoparticles to the animals with cardiac ischemiareperfusion injury was associated with a significant increase in myocardial silicon content in comparison with the sham-operated animals $(125 \pm 28.2$ versus $32 \pm 2.8 \mu \mathrm{g} / \mathrm{g}$, respectively, $P<0.05)$. Thus, the first evidence that nonmodified silica nanoparticles can accumulate within the anatomical area at risk after regional myocardial ischemiareperfusion was provided in this study.

\section{Effects of silica nanoparticle-bound and free adenosine on blood pressure and infarct size}

The data for mean blood pressure values are shown in Table 1. The extent of the blood pressure decrease was significantly less marked when adenosine was immobilized on the surface of the nanoparticles (Figure 5). The final series of experiments focused on investigation of the effect of free adenosine and nanoparticle-bound adenosine on infarct size. There were no differences in size of the risk area between the groups (Figure 6). Adenosine infusion caused a significant reduction in infarct size as compared with controls ( $29 \pm 6.2$ versus $63 \% \pm 7.3 \%$, respectively, $P<0.01$ ). Administration of adenosine adsorbed onto the silica nanoparticles resulted in a further significant decrease in infarct size in comparison with free adenosine (18 \pm 4.2 versus $29 \pm 6.2 \%$, respectively, $P<0.05$ ).

\section{Discussion}

The major finding of this study is that adsorption of adenosine onto silica nanoparticles enables passive delivery of adenosine to the ischemic-reperfused area of the heart, evidenced by more pronounced infarct size limitation and 
Table I Mean blood pressure in the course of infusion of free adenosine and silica nanoparticle-bound adenosine

\begin{tabular}{llllll}
\hline $\begin{array}{l}\text { Mean blood pressure, } \\
\mathbf{m m H g}\end{array}$ & Baseline & $\mathbf{5}$ min infusion & $\mathbf{1 0}$ min infusion & $\mathbf{5}$ min recovery & $\mathbf{1 0}$ min recovery \\
\hline Free ADO & & & & & \\
ADO + SNP & $125 \pm 12$ & $82 \pm 7$ & $85 \pm 9$ & $128 \pm 5$ & $119 \pm 13$ \\
\hline
\end{tabular}

Notes: Values are means \pm standard deviation $(\mathrm{SD})$. $* P<0.05$ vs free adenosine.

Abbreviations: ADO, adenosine; SNP, silica nanoparticles.

attenuation of the hemodynamic side effects typical of the drug used.

Several groups of drugs with different mechanisms of action might potentially be targeted to the heart using nanosized carriers. G protein-coupled receptor agonists (eg, adenosine, bradykinin, opioids), activators of tyrosine kinase receptors (insulin, insulin-like growth factor-1, transforming growth factor- $\beta 1$, erythropoietin), inhibitors of mitochondrial permeability transition pore (cyclosporine), and openers of ATP-sensitive potassium channels (nicorandil) are among the drugs used for pharmacological postconditioning in experimental and/or clinical studies. However, widespread clinical use of these drugs at doses required for a cardioprotective response is hampered by the high risk of dangerous side effects. For example, intravenous administration of adenosine is associated with arterial hypotension and bradycardia, ${ }^{20}$ while administration of bradykinin might lead to hypotension and bronchospasm. ${ }^{21}$ Cyclosporine, a mitochondrial permeability transition pore inhibitor, has been successfully used for pharmacological postconditioning in patients with acute ST elevation myocardial infarction..$^{22}$ Cyclosporine is commonly used for immune suppression, and its prolonged use is associated with several side effects, including renal failure, hepatotoxicity, infection, and increased risk of tumor development. Thus, it is of prime importance to develop the technique of targeted delivery of cyclosporine to the ischemic heart which may help to attenuate its systemic toxicity.

At present, some experimental data are available on targeting the heart using organic nanocarriers. In particular, antimyosin monoclonal antibody-doped liposomes containing ATP were administered to the isolated rat heart prior to global ischemia-reperfusion, ${ }^{11}$ which was associated with better postischemic contractile recovery. Intracoronary infusion of nontargeted ATP-loaded liposomes before regional ischemia-reperfusion injury resulted in a significant reduction in infarct size as compared with controls in a rabbit model. ${ }^{23}$ One possible limitation of these studies is that drug-loaded nanocarriers were administered prior to ischemia instead of being administered at the end of ischemia or at the early stage of reperfusion. It seems justified that accumulation of nanoparticles in the myocardium is most intensive at the time of reperfusion. Therefore, one can also expect to achieve optimal myocardial protection when infusing drug-loaded nanoparticles at this time. There are several lines of evidence in support of this notion. First, myocyte injury associated with exposure/expression of injury markers required for active targeting becomes more pronounced after reperfusion. Second, myocardial reperfusion initiates the inflammatory response associated with increased microvascular permeability, the latter being a major prerequisite for passive targeting. ${ }^{24}$ Third, reperfusion is accompanied by reactive hyperemia, which may contribute to better delivery of nanocarriers to the target area. ${ }^{25}$ Fourth, the early stage of reperfusion itself contributes strongly to the development of irreversible myocardial injury. ${ }^{2}$ Hence, release of the nanoparticle payload during this narrow time interval may prevent or block some of the mechanisms of lethal reperfusion injury. Thus, in order to optimize the pharmacokinetic profile of a heart-targeted drug delivery system, it should be administered at the final stages of ischemia or at the very beginning of reperfusion.

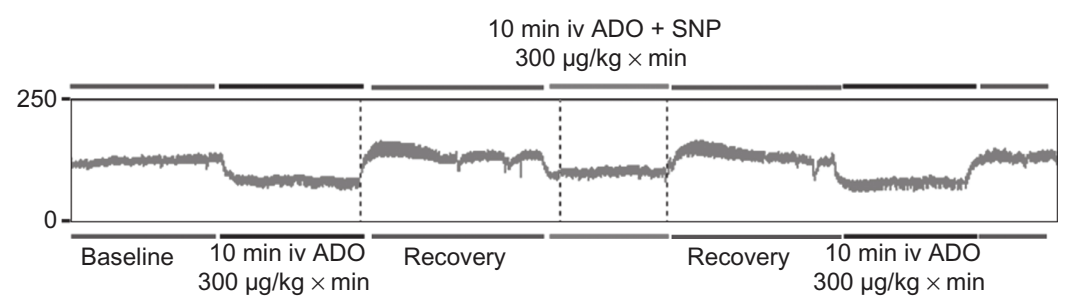

Figure 5 Representative blood pressure recording showing different blood pressure response to free and silica nanoparticle-bound adenosine. Initial free adenosine infusion was followed by recovery period and infusion of silica nanoparticle-bound adenosine. After recovery, one more episode of adenosine infusion was performed. Abbreviations: ADO, adenosine; SNP, silica nanoparticles. 


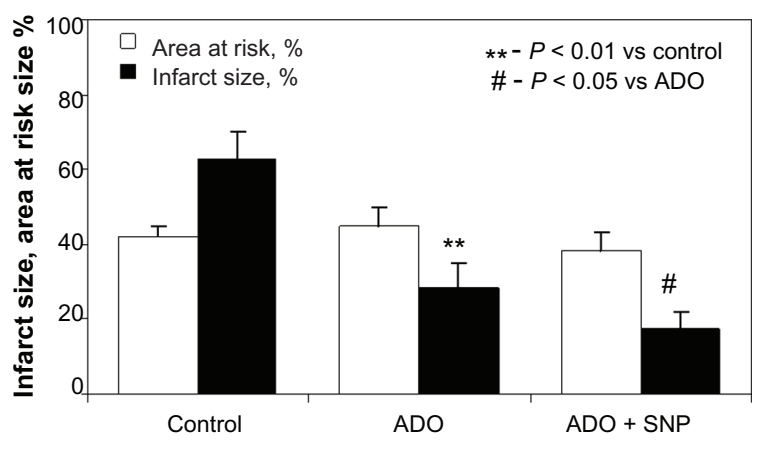

Figure 6 Area at risk size and infarct size in experimental groups. Abbreviations: ADO, adenosine; SNP, silica nanoparticles.

In the study reported by Scott et al, ${ }^{10}$ vascular endothelial growth factor-loaded liposomes functionalized with monoclonal antibodies against $\mathrm{P}$-selectin were administered intravenously to rats after permanent coronary artery ligation. The investigators showed increased capillary density within the infarct area 4 weeks after infarction and better post-infarct left ventricular function. Despite these positive findings, it is difficult to extrapolate the results of this study to current clinical practice because it was performed using a permanent ischemia model without reperfusion. At the same time, the current gold standard for treatment of acute coronary syndrome is prompt myocardial revascularization. Further, the benefits of this treatment regimen might be explained more by improved neovascularization of the peri-infarct area, and not by direct prevention of cardiac myocyte death within the ischemic area.

Important results were obtained in a landmark study by Takahama et $\mathrm{al}^{20}$ who used liposomal adenosine for treatment of myocardial ischemia-reperfusion injury in rats. Adenosine-loaded liposomes were infused intravenously for 10 minutes, starting 5 minutes prior to myocardial reperfusion. Selective accumulation of liposomes within the ischemic-reperfused area was confirmed using electron microscopy, optical fluorescence, and radionuclide imaging. In addition, liposomal adenosine administration was associated with more significant infarct size limitation and less severe arterial hypotension as compared with treatment using free adenosine. Taken together, our data are in agreement with these results. At the same time, there are several major differences in the characteristics of the carrier. In our study, we demonstrated for the first time that inorganic nanoparticles with a small diameter of 6-13 nm could be used for targeted drug delivery to the ischemic-reperfused heart. There are several potential advantages of silica nanoparticles compared with other drug nanocarriers. Different strategies for drug immobilization on the surface of the silica nanoparticles could be used, which may predetermine the different pattern of release required for optimal effect. There are at least three known methods, ie, covalent binding of the drug to the functional group of the spacer molecule engrafted on the surface of the functionalized silica, ionic immobilization, and physical adsorption. Further, several drugs with different mechanism(s) of action and distinct chemical structure can be immobilized on the surface of the silica nanoparticles at varying ratios. Lastly, silica nanomaterials are relatively inexpensive, with a long history of successful chemical modification and functionalization. ${ }^{26}$

An important question related to the potential use of silica nanoparticles in medical applications is particle toxicity. In cell culture experiments, mesoporous silica nanoparticles have been shown to have no injurious effect on viability and plasma membrane integrity, ${ }^{26}$ which is consistent with our acute experiments in vivo. ${ }^{12}$ With regard to long-term trials, the toxicity issue remains controversial. Kumar et $\mathrm{al}^{27}$ in nude mice experiments showed complete clearance of organically modified $20-25 \mathrm{~nm}$ silica nanoparticles from the organism via hepatobiliary excretion within 15 days after a single intravenous infusion, with no sign of organ toxicity. In contrast, Xie et al, ${ }^{28}$ in a partially analogous experimental setting, demonstrated extensive liver injury, ie, hepatocyte necrosis and mononuclear infiltration, accompanied by retention of the silica nanoparticles in the reticuloendothelial system for over 30 days. The hepatotoxic effect after either single or repeated silica nanoparticle administration has also been reported by others. ${ }^{29,30}$ Differences in hepatotoxic effects, apart from other factors, might be accounted for by the distinct characteristics of particle size $\mathrm{e}^{31}$ or surface charge, ${ }^{32}$ or both. Noteworthy, in our study, the liver silicon content in the silica nanoparticle-treated animals was documented to be at least six-fold higher than in the controls for up to 30 days after administration. Therefore, it seems that further experimentation is required to optimize both the dosage and physicochemical characteristics which can potentially provide better biodegradability.

In conclusion, silica nanoparticles are inert materials that might be used as carriers for heart-targeted drug delivery. Immobilization of adenosine on the surface of silica nanoparticles resulted in enhancement of free adenosinemediated infarct size limitation in an animal model. Further, the hypotensive effect of adenosine was attenuated after its adsorption on silica nanoparticles. The technique of active adenosine targeting will be studied to identify an approach that might be potentially helpful in clinical practice. 


\section{Disclosure}

The authors report no conflicts of interest in this work.

\section{References}

1. Sanada S, Komuro I, Kitakaze M. Pathophysiology of myocardial reperfusion injury: preconditioning, postconditioning, and translational aspects of protective measures. Am J Physiol Heart Circ Physiol. 2011;301(5):H1723-H1741.

2. Yellon DM, Hausenloy DJ. Myocardial reperfusion injury. NEngl J Med. 2007;357(11):1121-1135.

3. Galagudza M, Kurapeev D, Minasian S, Valen G, Vaage J. Ischemic postconditioning: brief ischemia during reperfusion converts persistent ventricular fibrillation into regular rhythm. Eur J Cardiothorac Surg. 2004;25(6):1006-1010.

4. Zhao ZQ. Postconditioning in reperfusion injury: a status report. Cardiovasc Drugs Ther. 2010;24(3):265-279.

5. Galagudza MM, Blokhin IO, Shmonin AA, Mischenko KA. Reduction of myocardial ischemia-reperfusion injury with pre- and postconditioning: molecular mechanisms and therapeutic targets. Cardiovasc Hematol Disord Drug Targets. 2008;8(1):47-65.

6. Ye X, Yang D. Recent advances in biological strategies for targeted drug delivery. Cardiovasc Hematol Disord Drug Targets. 2009;9(3): 206-221.

7. Matsumura Y, Maeda H. A new concept for macromolecular therapeutics in cancer chemotherapy: mechanism of tumoritropic accumulation of proteins and the antitumor agent SMANCS. Cancer Res. 1986;46(12 Pt 1):6387-6392.

8. Arias JL, Clares B, Morales ME, Gallardo V, Ruiz MA. Lipid-based drug delivery systems for cancer treatment. Curr Drug Targets. 2011;12(8):1151-1165.

9. Hirsjarvi S, Passirani C, Benoit JP. Passive and active tumour targeting with nanocarriers. Curr Drug Discov Technol. 2011;8(3):188-196.

10. Scott RC, Rosano JM, Ivanov Z, et al. Targeting VEGF-encapsulated immunoliposomes to MI heart improves vascularity and cardiac function. FASEB J. 2009;23(10):3361-3367.

11. Verma DD, Levchenko TS, Bernstein EA, Mongayt D, Torchilin VP. ATP-loaded immunoliposomes specific for cardiac myosin provide improved protection of the mechanical functions of myocardium from global ischemia in an isolated rat heart model. J Drug Target. 2006;14(5):273-280.

12. Galagudza MM, Korolev DV, Sonin DL, et al. Targeted drug delivery into reversibly injured myocardium with silica nanoparticles: surface functionalization, natural biodistribution, and acute toxicity. Int J Nanomedicine. 2010;5:231-237.

13. Donato M, D'Annunzio V, Berg G, et al. Ischemic postconditioning reduces infarct size by activation of A1 receptors and $\mathrm{K}+(\mathrm{ATP})$ channels in both normal and hypercholesterolemic rabbits. J Cardiovasc Pharmacol. 2007;49(5):287-292.

14. Xi L, Das A, Zhao ZQ, et al. Loss of myocardial ischemic postconditioning in adenosine $\mathrm{A} 1$ and bradykinin $\mathrm{B} 2$ receptors gene knockout mice. Circulation. 2008;118(14):S32-S37.

15. Morrison RR, Tan XL, Ledent C, Mustafa SJ, Hofmann PA. Targeted deletion of A2A adenosine receptors attenuates the protective effects of myocardial postconditioning. Am J Physiol Heart Circ Physiol. 2007;293(4):H2523-H2529.

International Journal of Nanomedicine

\section{Publish your work in this journal}

The International Journal of Nanomedicine is an international, peerreviewed journal focusing on the application of nanotechnology in diagnostics, therapeutics, and drug delivery systems throughout the biomedical field. This journal is indexed on PubMed Central, MedLine, CAS, SciSearch ${ }^{\circledR}$, Current Contents ${ }^{\circledR} /$ Clinical Medicine,
16. Xi J, McIntosh R, Shen X, et al. Adenosine A2A and A2B receptors work in concert to induce a strong protection against reperfusion injury in rat hearts. J Mol Cell Cardiol. 2009;47(5):684-690.

17. Norton ED, Jackson EK, Turner MB, Virmani R, Forman MB. The effects of intravenous infusions of selective adenosine A1-receptor and A2-receptor agonists on myocardial reperfusion injury. Am Heart $J$. 1992;123(2):332-338.

18. Pantely GA, Bristow JD. Adenosine. Renewed interest in an old drug. Circulation. 1990;82(5):1854-1856.

19. Finnie KS, Waller DJ, Perret FL, et al. Biodegradability of sol-gel silica microparticles for drug delivery. J Sol-Gel Sci Technol. 2009;49: $12-18$.

20. Takahama H, Minamino T, Asanuma H, et al. Prolonged targeting of ischemic/reperfused myocardium by liposomal adenosine augments cardioprotection in rats. J Am Coll Cardiol. 2009;53(8):709-717.

21. Homma T, Irvin CG. Bradykinin-induced bronchospasm in the rat in vivo: a role for nitric oxide modulation. Eur Respir J. 1999;13(2): 313-320.

22. Piot $\mathrm{C}$, Croisille $\mathrm{P}$, Staat $\mathrm{P}$, et al. Effect of cyclosporine on reperfusion injury in acute myocardial infarction. $N$ Engl J Med. 2008;359(5): 473-481.

23. Verma DD, Hartner WC, Levchenko TS, Bernstein EA, Torchilin VP. ATP-loaded liposomes effectively protect the myocardium in rabbits with an acute experimental myocardial infarction. Pharm Res. 2005;22(12):2115-2120.

24. Frangogiannis NG, Smith CW, Entman ML. The inflammatory response in myocardial infarction. Cardiovasc Res. 2002;53(1):31-47.

25. Okamura A, Ito $\mathrm{H}$, Iwakura $\mathrm{K}$, et al. Effect of reactive hyperemia after coronary recanalization on myocardial tissue reperfusion by thrombolysis in myocardial infarction flow grade in acute myocardial infarction. Am J Cardiol. 2006;97(5):617-623.

26. Slowing II, Vivero-Escoto JL, Wu CW, Lin VS. Mesoporous silica nanoparticles as controlled release drug delivery and gene transfection carriers. Adv Drug Deliv Rev. 2008;60(11):1278-1288.

27. Kumar R, Roy I, Ohulchanskky TY, et al. In vivo biodistribution and clearance studies using multimodal organically modified silica nanoparticles. ACS Nano. 2010;4(2):699-708.

28. Xie G, Sun J, Zhong G, Shi L, Zhang D. Biodistribution and toxicity of intravenously administered silica nanoparticles in mice. Arch Toxicol. 2010;84(3):183-190.

29. Nishimori H, Kondoh M, Isoda K, et al. Silica nanoparticles as hepatotoxicants. Eur J Pharm Biopharm. 2009;72(3):496-501.

30. Nishimori H, Kondoh M, Isoda K, et al. Histological analysis of 70-nm silica particles-induced chronic toxicity in mice. Eur J Pharm Biopharm. 2009;72(3):626-629.

31. Cho M, Cho WS, Choi M, et al. The impact of size on tissue distribution and elimination by single intravenous injection of silica nanoparticles. Toxicol Lett. 2009;189(3):177-183.

32. Isoda K, Hasezaki T, Kondoh M, Tsutsumi Y, Yagi K. Effect of surface charge on nano-sized silica particle-induced liver injury. Pharmazie. 2011;66(4):278-281.

Journal Citation Reports/Science Edition, EMBase, Scopus and the Elsevier Bibliographic databases. The manuscript management system is completely online and includes a very quick and fair peer-review system, which is all easy to use. Visit http://www.dovepress.com/ testimonials.php to read real quotes from published authors. 